\title{
Improved Early Stage (T1/2) Oral Tongue Cancer Medial Pathology Margins Using Horizontal Mattress Suture Technique
}

\author{
Jimmy Argo ${ }^{1}$, White Emily ${ }^{1}$, Brandon Pierson ${ }^{1}$, Elizabeth Gillies ${ }^{2}$, Michael Anderson ${ }^{3}$ and Nilesh Vasan*1 \\ ${ }^{1}$ University of Oklahoma Health Sciences Center Department of Otorhinolaryngology, Oklahoma, USA \\ ${ }^{2}$ University of Oklahoma Health Sciences Center Department of Pathology, Oklahoma, USA
}

${ }^{3}$ University of Oklahoma Health Sciences Center Department of Biostatistics, Oklahoma, USA

Received: November 10, 2017; Published: November 16, 2017

*Corresponding author: Nilesh Vasan, University of Oklahoma Health Sciences Center Department of Otorhinolaryngology, Oklahoma, USA, Email: Nilesh-Vasan@ouhsc.edu

\begin{abstract}
Objectives: Description of a novel surgical technique for partial glossectomy in oral tongue cancer patients with comparison of pathologic margins to conventional technique.

Methods: Patients with oral tongue cancer underwent partial glossectomy using a new horizontal mattress surgical technique developed by the senior author and were compared retrospectively to an age, sex and stage matched cohort. Anterior, posterior, and medial pathology margins were compared utilizing the student's t-test for normally distributed variables, and Wilcoxon-Mann-Whitney test for variables which were not normally distributed.
\end{abstract}

Results: 10 patients underwent partial glossectomy with the new technique. The mean medial pathology margin was significantly greater in the new technique group $(1.40 \mathrm{~cm}$ vs $0.88 \mathrm{~cm}, \mathrm{p}=0.04)$. There were no significant differences in anterior margin or posterior margin, age, tumor size or depth of invasion.

Conclusions: The new surgical technique using horizontal mattress sutures for dissection guidance and specimen orientation yielded improved medial pathology margins compared to conventional technique.

Keywords: Partial Glossectomy; Oral Tongue Cancer; Horizontal Mattress; Pathologic Margins; Novel Surgical Technique

\section{Introduction}

The oral tongue is the most common site for oral cavity cancers. Squamous cell carcinoma (SCCa) is responsible for over $90 \%$ of cases with an estimated incidence of 3.0/100,000 in the United States $[1,2]$. Males with a history of tobacco and alcohol use are at greatest risk and commonly present with early stage lesions. Treatment involves wide local excision, neck dissection if indicated and possibly post-operative radiation therapy depending on pathological features. The 5 year survival is $75-89 \%$ for early stage disease [2].

Unfortunately, locoregional recurrence is the most common cause of treatment failure. Inadequate surgical resection margins are often attributed to local recurrences while tumor depth, occult metastasis, and insufficient treatment of the neck contribute to regional recurrences. Locoregional recurrences of $23 \%$ have been reported in $\mathrm{T} 1 / \mathrm{T} 2$ oral tongue cancer patients with margins $<5 \mathrm{~mm}$ despite adjuvant therapy [3]. In contrast, local recurrence has been shown in only $11 \%$ with tumor free margins, showing that establishing adequate negative margins is imperative to preventing recurrence [2,4]. Operative success in terms of negative margins is largely determined by the surgeon's experience and frozen section analysis. Achieving clear pathologic margins is often challenging as distortion of the specimen during mobilization may compromise an adequate margin around the specimen. Although various techniques are utilized to remove tongue cancers, information is limited in regards to obtaining consistently acceptable surgical margins. We describe a novel technique to improve accuracy and consistency of tumor resection using horizontal mattress sutures.

\section{Patients and Methods}

Patients were prospectively selected between February 2011 and July 2013. All patients of the senior author (NV) with a diagnosis of $\mathrm{T} 1$ and $\mathrm{T} 2$ squamous cell carcinoma of the oral tongue were eligible for the study provided mattress sutures 
could be adequately applied around the lesion's resection margin. Patients with significant tumor extension to the base of tongue and floor of mouth were excluded as the sutures become difficult to insert. Approval for the study was obtained from the University of Oklahoma institutional review board. The operations were performed at the University of Oklahoma Health Sciences Center. Patients who underwent partial glossectomy with the new technique were compared with a cohort of patients who had undergone partial glossectomy with conventional technique i.e. no mattress sutures.

Patients were matched based on age, sex, and stage to a retrospective comparison cohort selected from prior tongue cancer patients of the department. Observations were classified into two groups: new suture technique and conventional technique. Comparisons of margins at the anterior, posterior, and medial aspects of the pathologic specimens were made between the groups. Mean values were compared with the student's t-test for normally distributed variables, and median values were compared with the Wilcoxon-Mann-Whitney test for variables which were not normally distributed. $\mathrm{P}<0.05$ was considered a significant result.

\section{Description of Procedure}

Depending on the extent of resection, a tracheostomy may be required to secure the airway before or after the resection as deemed necessary by the surgeon. The tumor is assessed both visually and by palpation after reviewing preoperative imaging studies. The tongue is tethered using 4-0 silk sutures around its anterior and lateral borders to maintain orientation and provide traction. A $1.5 \mathrm{~cm}$ margin5 is then marked around the lesion. A semi-circle is drawn from the posterolateral margin medially around the cancer and connected to the anterior margin on the dorsal surface. A similar line is drawn on the ventral surface and connected to dorsal markings at the lateral surfaces.

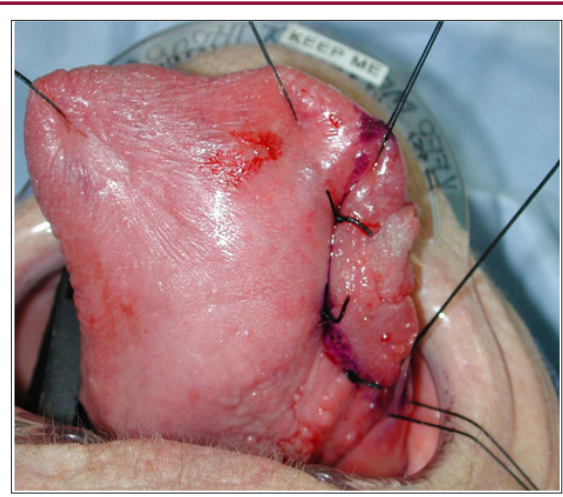

Figure 1: Small oral tongue cancer is prepared for excision using tethering sutures for adequate exposure and horizontal mattress sutures along an inked margin to define the margin of.

To maintain the plane of dissection and preserve margins, 2-0 silk horizontal mattress sutures are placed along the tongue markings in a sequential fashion usually using at CT1 curved needle. Each suture is placed through the dorsal and adjacent ventral surfaces, passed back through the tongue to the dorsal surface and tied. The concave aspect of the needle faces the cancer lesion to maximize suture distance away from the cancer. A suture scaffold is created to maintain normal anatomic position as the specimen is mobilized (Figure 1).

Anterior traction is applied using the tethering sutures for exposure. Resection is performed using electrocautery to dissect along the medial aspect of the mattress sutures. A surgical plane is maintained without undermining of surrounding normal tissue of either the specimen or the residual tongue (Figure 2). The mattress suture prevents tissue stretching and distortion during manipulation and maintains both specimen orientation and integrity for pathological analysis [5]. In most instances of early tongue cancer, the surgeon does not need to manually manipulate the tongue. The tumor is removed having uniform clean edges with the mattress sutures intact on the specimen. Following removal of the specimen, tissue is oriented for frozen section and transported to the pathologist with the mattress sutures intact. Tissue splaying and shrinkage of margin distance that occurs with retraction and manipulation are reduced by these sutures which also maintain anatomic integrity of the excised specimen (Figure 3 ).

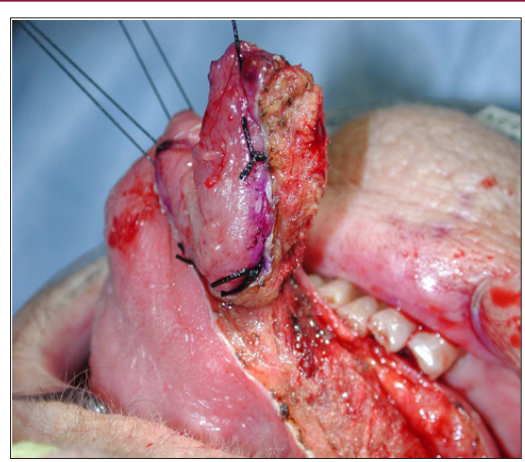

Figure 2: Oral tongue cancer is excised using the horizontal mattress sutures to define the surgical plane. Sutures remain intact on specimen side.

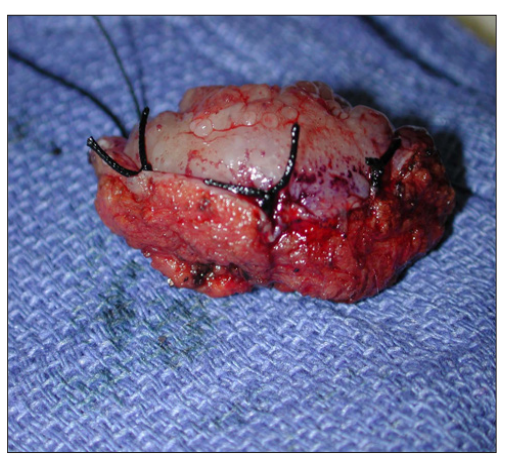

Figure 3: The specimen is removed and sent for frozen section analysis. Stretching and distortion of normal architecture are prevented by sutures.

\section{Results}

Between February 2010 and July 2013, a total of 10 patients underwent partial glossectomy utilizing the new suture technique. The mean age was 60.8 years old (range: $24-88$ ). The male to female ratio was equal at $1: 1$. The tumors were all $\mathrm{T} 1$ or $\mathrm{T} 2$ and ranged in size from 1.2 to $3.3 \mathrm{~cm}$. Sixty percent of the tumors were staged as pT1. The median depth of invasion was $0.65 \mathrm{~cm}$ and ranged from 0.4 to $1.6 \mathrm{~cm}$. Upon comparing the two groups, there were no significant differences with regard to the mean tumor size $(1.91 \mathrm{~cm}$ 
vs. $1.71 \mathrm{~cm}, \mathrm{p}=0.67)$ and depth of invasion $(0.65 \mathrm{~cm}$ vs. $0.80 \mathrm{~cm}$, $\mathrm{p}=0.73$ ). The mean age was 60.8 years for the new technique group and 61.5 years for the conventional group ( $\mathrm{p}=0.93$ ).

The mean medial pathology margin however was significantly greater in the new suture technique group compared to the conventional group, $1.40 \mathrm{~cm}$ vs $0.88 \mathrm{~cm}$ respectively ( $\mathrm{p}=0.04)$. No significant differences were found between the two groups for either the mean anterior margin ( $1.10 \mathrm{~cm}$ vs. $1.25 \mathrm{~cm}, \mathrm{p}=0.79)$ or the mean posterior margin ( $1.47 \mathrm{~cm}$ vs. $1.13 \mathrm{~cm}, \mathrm{p}=0.15)$. Average tumor free margins were increased by $0.65 \mathrm{~cm}$ in the new suture technique group, although this difference was not found to be significant $(\mathrm{p}=.14)$.

\section{Discussion}

Adequate tumor resection is important for local disease control of oral tongue squamous cell carcinoma. Wide local excision of the tumor remains the primary treatment modality for this subsite as 5-year disease specific survival has been shown to be significantly lower when clear margins are not obtained (43\% vs 59\% for patients with pathologically clear margins) [6]. It has previously been shown that $84 \%$ of errors occurring during intra-operative consultation for margin adequacy were the result of incorrect sampling [7,8]. Also, repeat frozen sections impose potentially avoidable pathology expenses and operative fees to the patient. Our technique for partial glossectomy of early oral tongue cancers describes a simple approach to maintain consistency of pathologic margins and help improve accuracy. Tissue splaying and margin shrinkage are reduced as the horizontal mattress sutures preserve normal anatomic integrity and orientation (Figure 4).

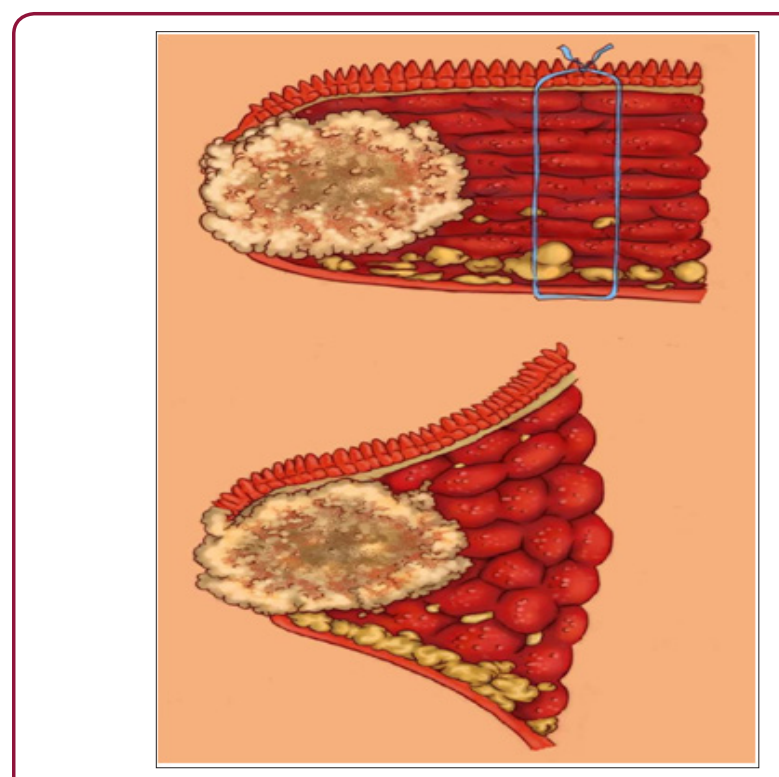

Figure 4: Tissue splaying and margin shrinkage are prevented by sutures following removal of the specimen.

This technique is quick and easy, especially for more anteriorly located oral tongue cancers. Larger lesions extending towards the tongue base can make placement of the sutures difficult however the senior author has used this method in T3 lesions including those that approach the tongue base. The technique is also easy to implement for surgeons in training as is the case in our practice. The scaffolding provides concrete landmarks to assist excision and prevent unnecessary manipulation and interruptions in the procedure to evaluate the surgical plane and margin. We have found that consistent margin distance is more frequently obtained using the horizontal mattress technique. We recognize that we have presented a small number of early stage cases; however, this is a reflection of our referred patient population which tends to be mainly those with advanced stage disease (T3/4) for which this technique has limited application i.e. composite resections with glossectomy for example. This study is also limited by the retrospective nature of the comparison group although they were age, sex, and stage matched. A prospective trial could be undertaken to confirm these results.

\section{Conclusion}

The new simple and easy to perform surgical technique described using horizontal mattress sutures for dissection guidance and specimen orientation increased significantly our medial pathology margins. Definitive wide margins may also allow the patient to avoid unnecessary adjuvant therapy such as postoperative radiation treatment to the primary site which can lead to significant morbidity.

\section{References}

1. Moore SR, Johnson NW, Pierce AM, Wilson DF (2000) the epidemiology of tongue cancer: a review of global incidence. Oral Diseases 6(2): 75-84.

2. Ganly I, Patel S, Shah J (2012) Early stage squamous cell cancer of the oral tongue-clinicopathologic features affecting outcomes. Cancer 118(1): 101-111.

3. Brennan S, Corry J, Kleid S, Porceddu S, Yuen K, et al. (2010) Prospective trial to evaluate staged neck dissection or elective neck readiotherapy in patients with CT- staged T1-2 N0 squamous cell carcinoma of the oral tongue. Head Neck 32(2): 191-198.

4. Wolfensberger M, Zbaeren P, Dulguerov P, Muller W, Arnoux A, et al. (2001) surgical treatment of early oral carcinoma-results of a prospective controlled multicenter study. Head Neck 23(7): 525-530.

5. Yuen PW, Lam KY, Chan ACL, Wei WI, Lam LK (1998) Clinicopathological analysis of local spread of carcinoma of tongue. Am J Surg 175(3): 242244.

6. Sessions DG, Spector GJ, Lenox J, Haughey B, Chao C, et al. (2002) Analysis of treatment results for oral tongue cancer. Laryngoscope 112(4): 616625.

7. Laurence J, Lin J, Karageorge LS, Powers CN (2000) Accuracy, utility, and cost of frozen section margins in head and neck cancer surgery. Laryngoscope 110(1): 1773-1776.

8. Grandeur Edwards R, Donald PJ, Wiese D (1993) the accuracy and clinical utility of frozen section diagnosis in head and neck surgery Experience at a university medical center. Head Neck 15: 33-38. 


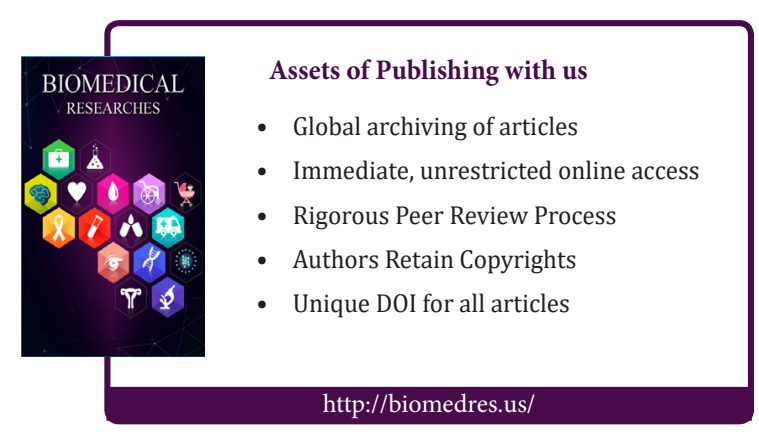

